\title{
Mixing Rules Formulation for a Kinetic Model of the Langmuir-Hinshelwood Semipredictive Type Applied to the Heterogeneous Photocatalytic Degradation of Multicomponent Mixtures
}

\author{
John Wilman Rodriguez-Acosta, ${ }^{1}$ Miguel Ángel Mueses, ${ }^{2}$ \\ and Fiderman Machuca-Martínez ${ }^{1}$ \\ ${ }^{1}$ Gaox Research Group, Chemical Engineering School, Universidad del Valle, A.A. 25360 Cali, Colombia \\ ${ }^{2}$ Photocatalysis and Solar Photoreactors Engineering, Department of Chemical Engineering, Universidad de Cartagena, \\ Postal 195, A.A. 1382 Cartagena, Colombia
}

Correspondence should be addressed to Fiderman Machuca-Martínez; fiderman.machuca@correounivalle.edu.co

Received 8 July 2014; Revised 15 September 2014; Accepted 18 September 2014; Published 16 October 2014

Academic Editor: Elisa Isabel Garcia-Lopez

Copyright ( 2014 John Wilman Rodriguez-Acosta et al. This is an open access article distributed under the Creative Commons Attribution License, which permits unrestricted use, distribution, and reproduction in any medium, provided the original work is properly cited.

\begin{abstract}
Mixing rules coupled to a semipredictive kinetic model of the Langmuir-Hinshelwood type were proposed to determine the behavior of the heterogeneous solar photodegradation with $\mathrm{TiO}_{2}-\mathrm{P} 25$ of multicomponent mixtures at pilot scale. The kinetic expressions were expressed in terms of the effective concentration of total organic carbon $\left(x_{\mathrm{TOC}}\right)$. An expression was obtained in a generalized form which is a function of the mixing rules as a product of a global contribution of the reaction rate constant $k^{\prime}$ and a mixing function $f(C)$. Kinetic parameters of the model were obtained using the Nelder and Mead (N-M) algorithm. The kinetic model was validated with experimental data obtained from the degradation of binary mixtures of chlorinated compounds (DCA: dichloroacetic acid and 4-CP: 4-chlorophenol) at different initial global concentration, using a CPC reactor at pilot scale. A simplex-lattice $\{2,3\}$ design experiment was adopted to perform the runs.
\end{abstract}

\section{Introduction}

Solar photocatalysis has been implemented and evaluated in the pollutant treatment at pilot scale in heterogeneous reactors using $\mathrm{TiO}_{2}-\mathrm{P} 25$, among which the following are highlighted: methylene blue [1], dichloroacetic acid, chlorinated compounds mixtures, phenol [2], 4-chlorophenol [3], dyes [4], pesticides and herbicides [5-9], and emerging contaminants [10]. Industrially, this technology has been applied to the treatment of polluted waters with lithographic dyes from the washing process of printing rollers used in a multinational company from Colombia. This solar plant of photocatalysis was one of the first commercial plants used in Latinoamerica [6]. Studies developed on these systems have demonstrated the technical and economical feasibility of their application as a clean technology in the treatment of pollutants on a large scale.

Scaling of these oxidation processes requires the inclusion of multiple variables that affect the reaction rate such as substrate concentration, catalyst concentration, $\mathrm{pH}$, radiation power, reactor geometry, and the presence of multiple reactive species among others. It is possible to find in several studies the effect of these variables on the process of photocatalytic oxidation of pure components [7, 11-13]; however, studies performed on the effect of the presence of multiple reactive species on the photooxidative process are limited.

Recently, Moulis and Krýsa [14] investigated the photodegradation of $n$-hexane, $n$-butyl acetate, toluene, and mixtures of two first ones, using $\mathrm{TiO}_{2}$ supported on plates and UV radiation in a continuous recycling system. It was found 
that the acetate is oxidized in higher proportion that the hexane when they are mixed, despite having similar kinetics.

Mueses et al. [2] used binary mixtures of DCA, phenol, and 4-chlorophenol in addition to the individual degradation of other organic pollutants in the validation of a generalized mathematic model for the degradation of pollutants in aqueous solution. Such a model was developed through the coupling of a modified equation for the reaction rate of $\mathrm{L}-\mathrm{H}$ type and a new model of effective quantum yield. Authors used a solar reactor of compound parabolic collectors (CPC) at pilot scale and catalyzed by $\mathrm{TiO}_{2}-\mathrm{P} 25$. The results revealed the high effectiveness of the model.

Mazille et al. [15] analyzed the performance of a catalyst of $\mathrm{TiO}_{2}$ with iron oxide immobilized on functionalized polyvinyl fluoride films (PVFf-TiO $-\mathrm{Fe}$ oxide) in the degradation mixture of pesticides, phenol, and nalidixic acid among others. Authors used a CPC reactor with UV radiation. The results were favorable, but less so for the pesticide mixture.

Colina-Márquez et al. [5] evaluated the use of the absorption-scattering model, so-called Six-Flux Model (SFM) for the characterization of radiant field and subsequent prediction of photomineralization of pesticides mixtures using a solar CPC reactor. The authors used a L-H type kinetic expression modified with the Local-Volumetric-Rate-Photon -Absorption (LVRPA) and this equation was expressed in terms of the concentration of TOC. Reactions were catalyzed by $\mathrm{TiO}_{2}-\mathrm{P} 25$ and the results of the implementation of the SFM were satisfactory for sunny and cloudy days.

Research from Gora et al. [12] and Toepfer et al. [16] reports the analysis of the photocatalytic oxidation of mixtures of three herbicides (isoproturon, simazine, and propazine) using $\mathrm{TiO}_{2}$ and an annular reactor irradiated with $\mathrm{UV}$ lamps. Their results were analyzed in terms of $\mathrm{L}-\mathrm{H}$ reaction model. Authors observed that these deviate at high substrate concentration (higher than $1 \mathrm{mg} / \mathrm{L}$ ) caused by the formation of multilayers on the catalyst surface.

Studies to date on the photocatalytic degradation of mixtures of organic pollutants have not considered explicitly the application of mixing rules, which can facilitate the analysis of the photooxidative behavior of multicomponent systems, having the advantage of allowing the identification of synergistic and antagonistic effects.

This work proposes the implementation of mixing rules to combine the individual kinetic behavior of DCA and 4$\mathrm{CP}$ in a single kinetic expression to predict the global kinetic behavior of the binary mixture. The respective experimental validations were performed in a CPC reactor irradiated with solar light, varying the initial global concentration of the mixture and the proportion of the reagents.

\section{Methodology}

2.1. Modelling of Degradation Kinetics. Heterogeneous photocatalytic reaction kinetics can be expressed in a general form as follows [17-19]:

$$
-r=k f(C),
$$

where $r$ is the reaction rate, $k$ represents the global kinetic constant of the reaction, and $f(C)$ is a function of the concentration of species involved in the reaction. Normally this reaction rate is of the Langmuir-HinshelwoodHougen-Watson type $[7,17,18]$.

To apply (1) in photocatalytic processes is necessary to take into account the fact that $f(C)$ depends on the reactions involved and on the experimental conditions. Several expressions for this function have been reported in kinetic studies of pollutant photodegradation [2]. One of the most used expressions in the treatment of organics arises from the consideration of only two elementary reaction steps (adsorption and chemical reaction) [18]:

$$
f(C)=\frac{K C}{1+K C}
$$

where $K$ is the adsorption constant. Typically, an absence of homogeneous photochemical reaction (reaction without catalyst) is considered. Equation (2) is valid only for a unique experimental device under fixed experimental conditions (e.g., constant radiation on the reactor). This is because $k$ varies with the reactor geometry, radiation source, and operational conditions. This implies that the expression is not useful neither for comparison between reaction devices nor scaling [20].

The reaction rate can be represented by (3) which has been successfully used to represent the experimental behavior in different studies of heterogeneous photocatalytic degradation [11, 14, 21-28]. In such studies, the influence of operating variables on the photooxidative process has been studied:

$$
-r=k \frac{K C}{1+K C}
$$

Studies that consider the effect of radiant field on the kinetics of photocatalytic degradation in models of $\mathrm{L}-\mathrm{H}$ type have been reported by several authors [5, 29-32].

Equation (3) is coupled to the material balance of the reactor to reproduce the experimental information. In the case of mixtures, the pollutants can be considered as a pseudocomponent; therefore the use of the variable "total organic carbon concentration (TOC)" facilitates the analysis and comparison of the experimental data with predictions performed by the kinetic model proposed here.

The mineralization process is a multistage process with multiple intermediate species. Using the TOC to analyze the overall performance of the process is a common practice; therefore it is possible to consider the modified reaction model as LH with a reasonable approximation, but in this case it should be applied to a pseudo compound [2, 4, 33].

The complexity of the degradation pathway of any compound is proportional to the complexity of its chemical structure; therefore it is expected that 4-CP presents the most complex route to complete mineralization than DCA. The reaction mechanism for the 4-CP is based on the electrophilic attack of the aromatic ring caused by adsorbed hydroxyl radicals (the indirect attack) $[34,35]$. However, for DCA, this is degraded by direct attack of the photogenerated holes $[33,34,36]$. 
Although the reaction mechanisms of the substances used are well known, this model assumes Langmuir-Hinshelwood behavior, without going into details of the reaction mechanism. So, if it use any reaction mechanism, the reaction rate expression can be changed as well as mixing rules.

2.1.1. Mixing Rules Formulation. From (1) the reaction rate expression is composed of two contributions $k$ and $f(C)$, which can be modified through the mixing rules to get a global kinetic expression that combines the kinetic contributions of each reagent which are generated in a system of multicomponent photocatalytic degradation.

Those mixing rules may extend to the use of the intrinsic kinetic parameters, since the effects of radiant field may be considered coupled to the "apparent" global constant of reaction rate as an invariant parameter; this means that (1) is in fact represented by several contributions:

$$
\begin{gathered}
-r=k^{\prime} f(C)=k f(C) f_{i}\left(F_{i}\right) \\
k^{\prime}=k f_{i}\left(F_{i}\right)
\end{gathered}
$$

where $k^{\prime}$ is the apparent global constant of reaction, in this case being equal to the constant $k$ in (3), and $f_{i}\left(F_{i}\right)$ is a function of the radiation reaching the reactor, which can be coupled to $k^{\prime}$ if this remains constant.

Therefore, when deducing a mixture expression to the contribution $k^{\prime}$ (not intrinsic) of the reaction rate, it may also apply to the contribution $k$ (intrinsic) if dependence of $r$ is uncoupled of the radiant field, which leads to the calculation of such a field.

The following assumptions were considered in the formulation of the mixture expressions. (i) The global reaction rate is the sum of the individual kinetics, this applies considering that the hydroxyl radicals generated during the photocatalytic degradation attack the organic matter in a nonselective way $[13,31]$. (ii) In the photocatalytic system the adsorption constants have a very low order of magnitude with respect to the reaction constant. Recently, this has been validated in the treatment of organic components by solar photocatalysis [2]. (iii) Constant radiant field, though the solar radiant field effects depend on the atmospheric fluctuations, can be considered as a constant function in the UV absorption range of the $\mathrm{TiO}_{2}-\mathrm{P} 25$ [2].

The first assumption (i) allows establishing an expression to the kinetic constant of the mixture $k_{m}$ applied to a binary system:

$$
\begin{aligned}
& \left(-r_{i}\right)+\left(-r_{j}\right)=k_{i} \frac{K_{i} \mathrm{TOC}_{i}}{1+K_{i} \mathrm{TOC}_{i}}+k_{j} \frac{K_{j} \mathrm{TOC}_{j}}{1+K_{j} \mathrm{TOC}_{j}}, \\
& \left(-r_{i}\right)+\left(-r_{j}\right) \\
& =\frac{k_{i} K_{i} \mathrm{TOC}_{i}+k_{j} K_{j} \mathrm{TOC}_{j}+2 K_{i} K_{j} \mathrm{TOC}_{i} \mathrm{TOC}_{j}}{1+K_{i} \mathrm{TOC}_{i}+K_{j} \mathrm{TOC}_{j}} .
\end{aligned}
$$

Considering assumption (ii), terms $K_{i} K_{j}$ have an order of magnitude relatively low ( $\ll 1)$ compared with the other terms of the equation and therefore they may be omitted:

$$
\left(-r_{A}\right)+\left(-r_{B}\right) \approx \frac{k_{i} K_{i} \mathrm{TOC}_{i}+k_{j} K_{j} \mathrm{TOC}_{j}}{1+K_{i} \mathrm{TOC}_{i}+K_{j} \mathrm{TOC}_{j}}
$$

An expression for the kinetic constant of the mixture $\bar{k}_{m}$ is obtained from the numerator of expression (6):

$$
\begin{gathered}
k_{i} K_{i} \mathrm{TOC}_{i}+k_{j} K_{j} \mathrm{TOC}_{j}=\bar{k}_{m}\left(K_{i} \mathrm{TOC}_{i}+K_{j} \mathrm{TOC}_{j}\right), \\
\bar{k}_{m}=\frac{k_{i} K_{i} \mathrm{TOC}_{i}+k_{j} K_{j} \mathrm{TOC}_{j}}{K_{i} \mathrm{TOC}_{i}+K_{j} \mathrm{TOC}_{j}}
\end{gathered}
$$

where subscript $m$ refers to the mixture.

In this work, we have considered defining the global fraction of TOC for component $i$, as $x_{i}$, given by

$$
x_{i}=\frac{\mathrm{TOC}_{i}}{\mathrm{TOC}_{m}},
$$

where $\mathrm{TOC}_{m}$ is the TOC concentration of the mixture. Using the $x_{i}$ fraction in (8) and generalizing to a multicomponent system, the following is obtained:

$$
\bar{k}_{m}=\frac{\sum_{i=1}^{n} k_{i} K_{i} x_{i}}{\sum_{i=1}^{n} K_{i} x_{i}},
$$

where $\bar{k}_{m}$ is the kinetic pseudoconstant of the reaction for the mixture, $k_{i}$ is the kinetic constant of the reaction of component $i, K_{i}$ is the adsorption constant for component $i$, and $n$ is the total number of components.

From the practical point of view, it is useful to use mass units such as ppm (TOC), considering it is an indirect measure of the mass of the individual compounds and mixtures during the mineralization process. Therefore the molar concentrations of the pollutants were not followed.

On the other hand, to obtain an expression to contribution $f(C)$ of the reaction rate in the case of the mixture, an analogy present between L-H model and one of the terms of the model proposed by Alfano et al. [37] was considered:

$$
\begin{aligned}
r_{p}=\alpha_{1}^{\prime}\left\{-\left(\frac{E\left(R_{i}, R_{j}\right)}{1+E\left(R_{i}, R_{j}\right)}\right)\right. & \\
& \left.+\sqrt{\left(\frac{E\left(R_{i}, R_{j}\right)}{1+E\left(R_{i}, R_{j}\right)}\right)^{2}+\frac{\alpha_{2}^{\prime}}{C_{m p} \int_{\lambda}^{\prime} e_{\lambda}^{a}(x, t) d \lambda}}\right\} \\
& \times \frac{\alpha_{3, i}\left[R_{i}\right]}{1+E\left(R_{i}, R_{j}\right)},
\end{aligned}
$$

wherein

$$
E\left(R_{i}, R_{j}\right)=\alpha_{3, i}\left[R_{i}\right]+\sum_{\substack{j=1 \\(j \neq i)}}^{n} \alpha_{3, j}\left[R_{j}\right],
$$


$r_{p}:$ reaction rate per particle, $\mathrm{mol} / \mathrm{s}$ particle,

$\alpha_{1}^{\prime}, \alpha_{2}^{\prime}$, and $\alpha_{3, i}$ : constants, $\mathrm{mol} / \mathrm{s}$ particle, g s/einstein, and $\mathrm{m}^{3} / \mathrm{mol}$, respectively,

$\left[R_{i}\right]$ : substrate concentration, $\mathrm{mol} / \mathrm{m}^{3}$,

$C_{m p}$ : mass concentration of catalyst, $\mathrm{g} / \mathrm{m}^{3}$,

$e_{\lambda}^{a}$ : local volumetric rate of radiation energy absorption, einstein $/ \mathrm{s} \mathrm{m}^{3}$.

Where $\left(E\left(R_{i}, R_{j}\right) /\left(1+E\left(R_{i}, R_{j}\right)\right)\right)$ is considered by the subsequent analysis. The variable $E\left(R_{i}, R_{j}\right)$ represents the pseudocomponent concentration, that is, the concentration of the mixture as a whole. This led to the approach of an expression for $f(C)$ in the case of the mixture given by

$$
f_{m}\left(C_{m}\right)=\frac{E\left(R_{i}, R_{j}\right)}{1+E\left(R_{i}, R_{j}\right)}
$$

Equation (13) may be expressed in terms of the TOC concentration obtaining

$$
f_{m}\left(C_{m}\right)=\frac{E\left(x, \mathrm{TOC}_{m}\right)}{1+E\left(x, \mathrm{TOC}_{m}\right)}=\frac{\mathrm{TOC}_{m} \sum_{i=1}^{n} K_{i} x_{i}}{1+\mathrm{TOC}_{m} \sum_{i=1}^{n} K_{i} x_{i}} .
$$

Because of the $x_{i}$ fraction changes during the course of the reaction and based on the first assumption it is possible to update such fraction using the models that describe the individual kinetics.

Finally, the kinetic expression of the mixture is given by the combination of the mixing rules (10) and (14) in the reaction rate equation:

$$
\begin{gathered}
-r_{m}=\bar{k}_{m} f_{m}\left(x, \mathrm{TOC}_{m}\right), \\
-r_{m}=\left(\frac{\sum_{i=1}^{n} k_{i} K_{i} x_{i}}{\sum_{i=1}^{n} K_{i} x_{i}}\right)\left[\frac{\mathrm{TOC}_{m} \sum_{i=1}^{n} K_{i} x_{i}}{1+\sum_{i=1}^{n}\left(K_{i} x_{i} \mathrm{TOC}_{m}\right)^{a}}\right],
\end{gathered}
$$

where exponent $a$ generalizes the form of the concentration term in the denominator of the kinetic expressions of the mixture components, being that, depending on the nature of the compound, this exponent could be different from the unit.

2.2. Material Balance and Reactor Modelling. The equation that describes the material balance in a solar heterogeneous photocatalytic reactor that uses this approach and coupling of the mixing rules is the following:

$$
V_{T} \frac{d\left(\mathrm{TOC}_{m}\right)}{d t_{30 \mathrm{~W}}}=-\left(\frac{\sum_{i=1}^{n} k_{i} K_{i} x_{i}}{\sum_{i=1}^{n} K_{i} x_{i}}\right)\left[\frac{\mathrm{TOC}_{m} \sum_{i=1}^{n} K_{i} x_{i}}{1+\sum_{i=1}^{n}\left(K_{i} x_{i} \mathrm{TOC}_{m}\right)^{a}}\right],
$$

where $V_{T}$ is the total volume of the reaction and $t_{30 \mathrm{~W}}$ is a standardized coordinate time, which is necessary to avoid the noise produced by the variations presented by the solar radiation and produced by the physical configuration of the reactor, taking into account the fact that the reactor consists of illuminated and nonilluminated parts that could give
TABLE 1: Simplex-lattice $\{2,3\}$ experimental design.

\begin{tabular}{lcc}
\hline Design points & \multicolumn{3}{c}{ Proportions } \\
\hline 1 & $4 \mathrm{CP}$ & DCA \\
2 & 1 & 0 \\
3 & $2 / 3$ & $1 / 3$ \\
4 & $1 / 3$ & $2 / 3$ \\
\hline
\end{tabular}

unreliable information if the experimental time is used as measure variable. This standardized time was proposed by Malato et al. [7] and is given by the next expression in the case of variable radiation intensity:

$$
t_{30 \mathrm{~W}}=\frac{1}{I_{\mathrm{G}, \mathrm{UV}}^{\circ}} \int_{0}^{t} I_{G, \mathrm{UV}}(t) d t
$$

wherein

$t_{30 \mathrm{~W}}$ : sample time at constant radiation of $30 \mathrm{~W} / \mathrm{m}^{2}$, $I_{G, U V}$ : instantaneous experimental $\mathrm{UV}$ radiation intensity, $\mathrm{W} / \mathrm{m}^{2}$,

$I_{G, \mathrm{UV}}^{\circ}$ : standard UV radiation intensity, equal to $30 \mathrm{~W} /$ $\mathrm{m}^{2}$.

\subsection{Experimental Validation}

2.3.1. Materials and Equipment. Dichloroacetic acid (Merck, 99.99\%), 4-chlorophenol (Merck, 99.99\%), and titanium dioxide (Degussa P-25) were used.

Tracking of the substrate concentration was followed using a total carbon analyzer (5050 TOC-VCPHSHIMADZU). The dissolved oxygen was measured with an ORION 083010MD electrode. The $\mathrm{pH}$ was measured with a multiparameter (Thermo Scientific Orion 5-Star Plus) using a probe with reference 910500 . The UV radiation intensity was monitored using a radiometer (ACADUS 85-PLS, 300$400 \mathrm{~nm}$ ).

The experiments were carried out in the solar laboratory of the Universidad del Valle (Cali-Colombia). Radiation intensity was set to $30 \mathrm{~W} / \mathrm{m}^{2}$ due to the standardization of the experimental time by using $t_{30 \mathrm{w}}$. The details of the experimental device are found in Colina-Márquez et al. [5].

2.4. Experimental Design. A simplex-lattice $\{2,3\}$ [38] experimental design was followed to assay the mixing rules proposed here, which sets the amount of experiments and the reagent proportions in the binary mixture. Such design represents proportion of $0,1 / 3,2 / 3$, and 1 for each reagent at a global concentration or a fixed substrate concentration. 3 substrate concentrations equal to 30,60 , and 120 ppm with 4 experiments per concentration (see Table 1) were considered; this produced a total of 12 experiments.

2.5. Photocatalytic Tests. The synthetic preparation of mixtures of DCA and 4-CP was performed using a volume of $100 \mathrm{~mL}$ of tap water (to emulate real operating conditions 


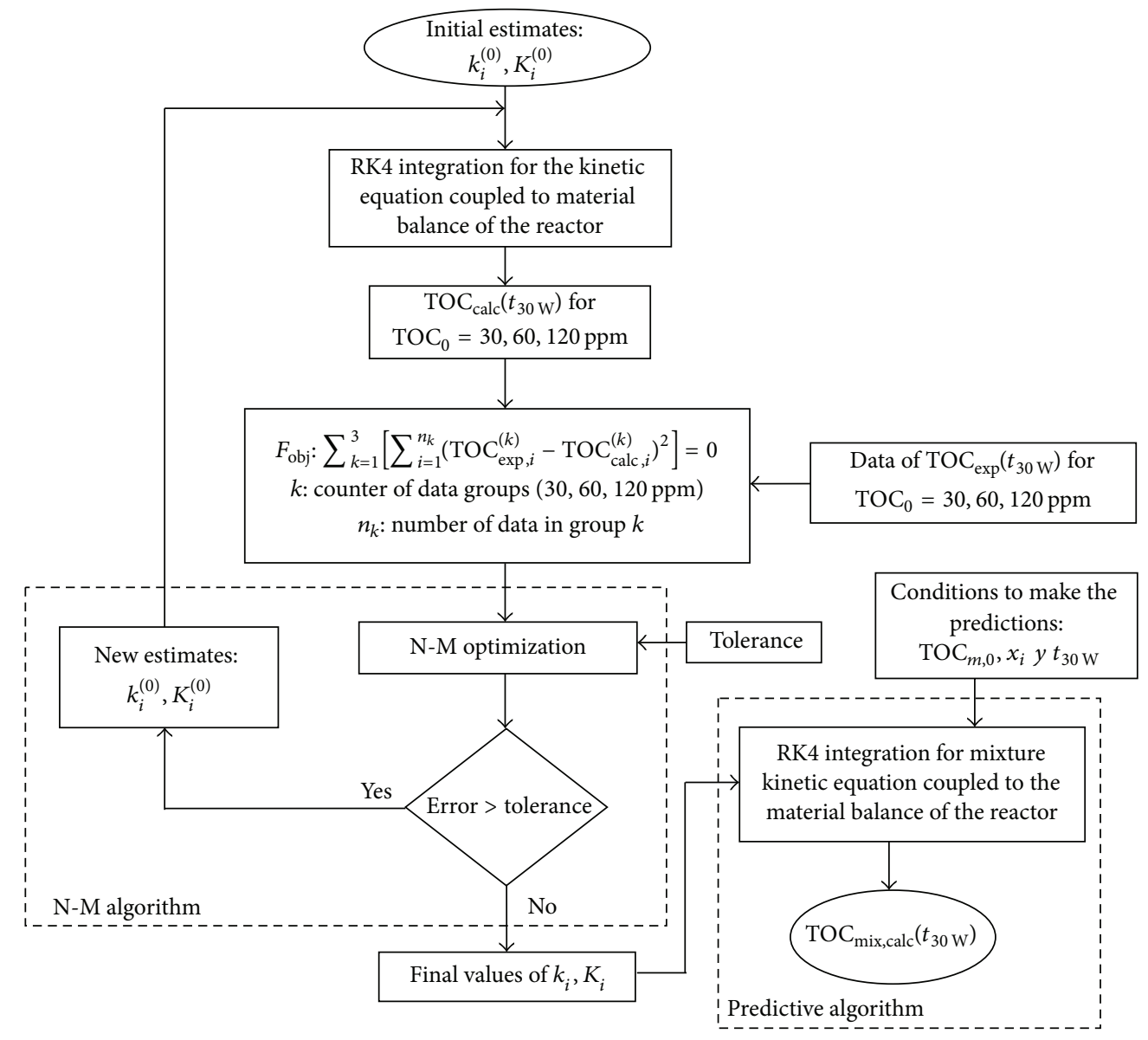

FIGURE 1: Algorithms of fitting of kinetic parameters and prediction by mixing model.

in industrial processing), following the scheme of global concentration given by the adopted experimental design.

The experimental procedure is detailed in Mueses et al. [2]. Concentration of catalyst was set to $0.3 \mathrm{~g} / \mathrm{L}$ based on studies performed by the previous researchers. Total reaction volume was set to $20 \mathrm{~L}$. The contributions of photolysis and molecular adsorption on the photocatalytic degradation of DCA and 4-CP were not significant as reported by Mueses et al. [2].

2.6. Evaluation of Kinetic Parameters. The fitting algorithm for obtaining the kinetic parameters was programmed in Matlab 7.0.4 using the ode45 routine which corresponds to fourth order Runge-Kutta (RK4) with adaptive step function and using the fminsearch which corresponds to the optimization algorithm of Nelder-Mead (N-M) [39]. The fitting and prediction algorithms are presented in Figure 1.

\section{Results and Discussion}

3.1. Prediction of Initial Reaction Rate. Applying the algorithm shown in Figure 1 allows evaluating the reaction rate at different conditions of initial concentration of substrate. Figure 2 shows the prediction of the model obtained for the initial reaction rates, where the dashed line represents the model behavior and the circles represent the three initial concentrations under study (30, 60, and 120 ppm). During the fitting of kinetic parameters exponent $a$ present in (16) was modified and it was observed that taking values of 1 for DCA and 2 for 4-CP a good experimental data representation was accomplished. The latter analyzed in terms of initial reaction rate shows that the DCA degradation behavior in the concentration range assessed tends to follow the behavior described by systems that involve two elemental reaction steps (adsorption reaction), as shown in [40].

Exponent $a$ from (16) may be different from the unit, considering the concentration in terms of TOC expresses the total quantity of pollutant that is present, leaving implicit on the reaction rate that is the sum of all contributions produced by intermediates of the reaction. Those contributions may affect the kinetic expression, especially in the adsorption term if they are significant. According to Gora et al. [12], in the photodegradation of some organic compounds, there may exist competition for active sites between the substrate and the reaction intermediates, which reduce the degradation of initial compound; in that case parameter $a$ would be greater than unit.

Likewise, Figure 3 shows the behavior obtained for 4$\mathrm{CP}$ which is similar to those involving complex reactions 


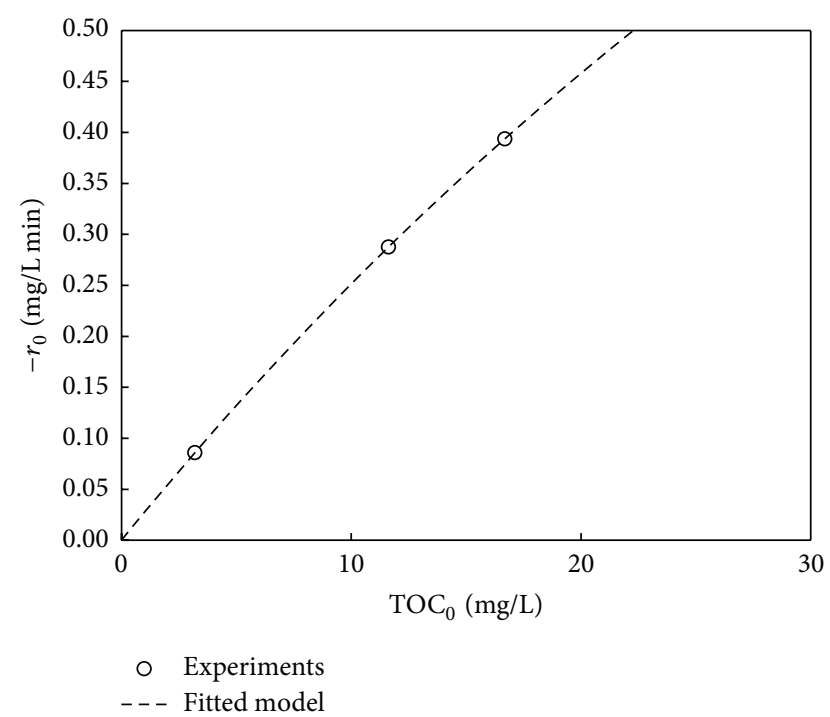

FIGURE 2: Initial concentration effect on the initial reaction rate of DCA.

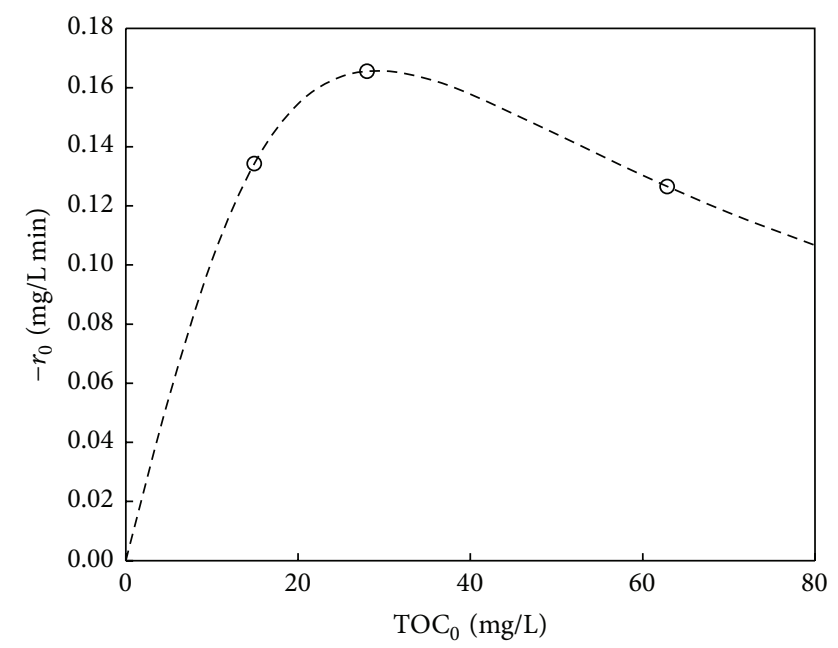

○ Experiments

- - - Fitted model

FIGURE 3: Initial concentration effect on the initial reaction rate of 4-CP.

(multistep reactions), as illustrated in [40]. This result is according to the reaction scheme proposed by Satuf et al. [35] to this compound, since 4-CP complies with the aforementioned.

3.2. Kinetic Expression for DCA. The kinetic expression for DCA coupled to the material balance for the reactor is shown in (18). Using the fitting algorithm presented in Figure 1 it was found that the values for $k$ and $K$ were $51.324 \mathrm{mg} / \mathrm{min}$ and $0.010859 \mathrm{~L} / \mathrm{mg}$, respectively. This equation showed

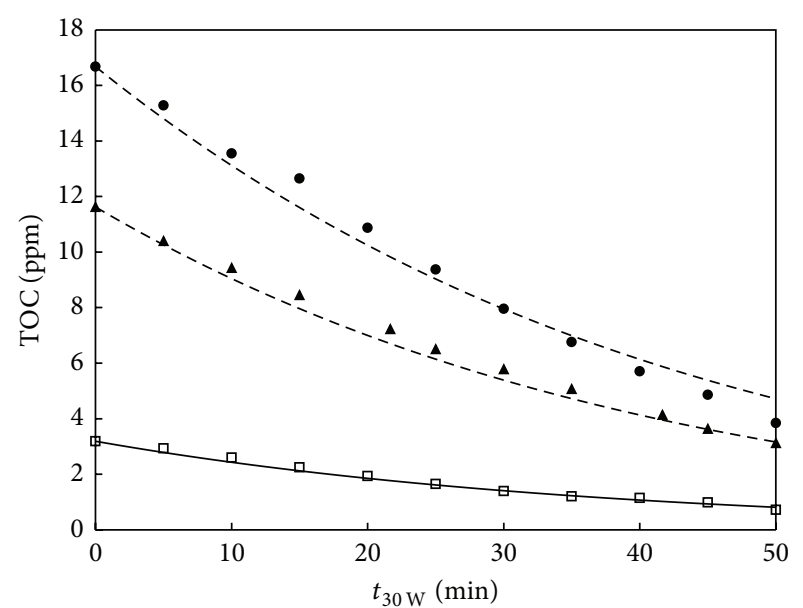

FIGURE 4: Experimental fitting of the selected model for the DCA: $30 \mathrm{ppm}(\square) ; 60 \mathrm{ppm}(\boldsymbol{\Delta}) ; 120 \mathrm{ppm}(\bullet)$.

a minimum $R^{2}$ of 0.983 and a maximum standard deviation of $0.60 \mathrm{ppm}$ :

$$
V_{T} \frac{d(\mathrm{TOC})}{d t_{30 \mathrm{~W}}}=-k \frac{K \mathrm{TOC}}{1+K \mathrm{TOC}} .
$$

Prediction of the experimental data with (18) is shown in Figure 4, wherein the markers represent experimental data and the lines the corresponding predictions.

According to Figure 4, the DCA tends to disappear quickly as the initial concentration is increased; this makes it a compound relatively easy to oxidize by photocatalysis and this is possible because that compound has a simple structure. This attribute may be more influential in the reactivity of the species during photodegradation than the one produced by the presence of complex or chelating groups [15].

3.3. Kinetic Expression for 4-CP. Equation (19) shows the kinetic expression for 4-CP coupled to material balance of the reactor. The values obtained for $k$ and $K$ were $6.62536 \mathrm{mg} / \mathrm{min}$ and $0.034268 \mathrm{~L} / \mathrm{mg}$, respectively. This equation showed a minimum $R^{2}$ of 0.88 and a maximum standard deviation calculated of 1.18 ppm:

$$
V_{T} \frac{d(\mathrm{TOC})}{d t_{30 \mathrm{~W}}}=-k \frac{K \mathrm{TOC}}{1+(\text { KTOC })^{2}} .
$$

Predictions made with (19) are shown in Figure 5. This figure shows that oxidation process for $4-\mathrm{CP}$ is slower if this is compared with DCA; this may be due to the complex route of degradation that is exposed by this compound.

3.4. Kinetic Model of Mixture for the System DCA-4-CP. Using parameters $k$ and $K$ from (18) and (19), in other words, the parameters for each reagent, in the expression that describes the material balance for the reactor in the mixture 


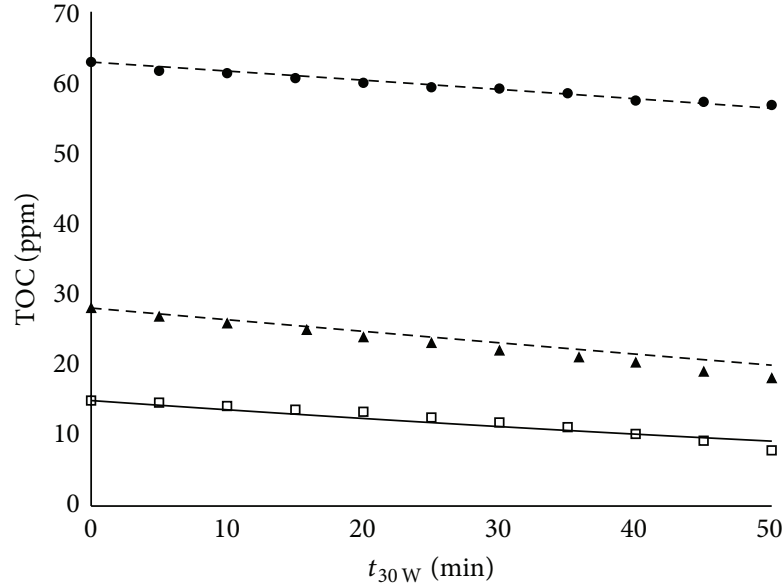

Figure 5: Experimental fitting of the modified model for 4-CP: 30 ppm (); 60 ppm (А); 120 ppm (•).

TABLE 2: Kinetic model parameters for the mixture.

\begin{tabular}{lcc}
\hline Parameter & Value & Unit \\
\hline$V_{T}$ & 20 & $\mathrm{l}$ \\
$k_{A}$ & 51.324 & $\mathrm{mg} / \mathrm{min}$ \\
$k_{B}$ & 6.625 & $\mathrm{mg} / \mathrm{min}$ \\
$K_{A}$ & 0.0108590 & $1 / \mathrm{mg}$ \\
$K_{B}$ & 0.0342685 & $1 / \mathrm{mg}$ \\
$x_{A 0}$ & $0.142-0.40$ & - \\
\hline
\end{tabular}

case, as shown in (16), it is possible to get (20) that represents the binary case:

$$
\begin{aligned}
V_{T} \frac{d\left(\mathrm{TOC}_{m}\right)}{d t_{30 \mathrm{~W}}} \\
=-\left[\frac{k_{A} K_{A} x_{A}+k_{B} K_{B}\left(1-x_{A}\right)}{K_{A} x_{A}+K_{B}\left(1-x_{A}\right)}\right] \\
\quad \times\left\{\frac{\mathrm{TOC}_{m}\left[K_{A} x_{A}+K_{B}\left(1-x_{A}\right)\right]}{1+K_{A} x_{A} \mathrm{TOC}_{m}+K_{B}^{2}\left(1-x_{A}\right)^{2} \mathrm{TOC}_{m}^{2}}\right\} .
\end{aligned}
$$

Subscripts $A$ and $B$ refer to DCA and 4-CP, respectively. Table 2 summarizes the used parameters in the behavior prediction of the binary mixtures photodegradation.

The prediction results performed at different initial concentrations of the mixture are shown in Figures 6, 7, and 8. In general, good predictions were observed on the mixture behavior, which is confirmed by the fitting statistical parameters (see Table 3).

The highest deviation was obtained in the mixture 1:2 (4CP:DCA), which is shown in Figure 6 that corresponds to a global concentration of $30 \mathrm{ppm}$. This fitting showed values of $R^{2}$ and $\sigma$ are equal to -0.628 and 2.58 , respectively. The deviation could suggest the occurrence of possible effects of mixing; in this case, they would correspond to a synergism between the components.

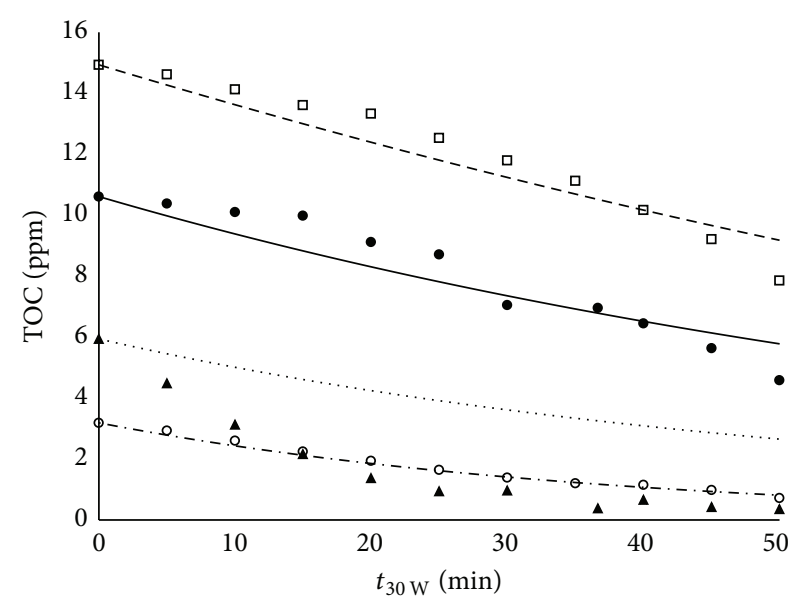

FIgURE 6: Solar mineralization of binary mixtures at $30 \mathrm{ppm}$.

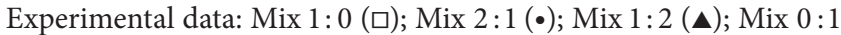
(○). Predictions: Mix 1:0 (---), Mix 2:1 (-), and Mix 1:2 (..), Mix $0: 1(--)$, wherein "Mix" represents 4-CP : DCA relation.

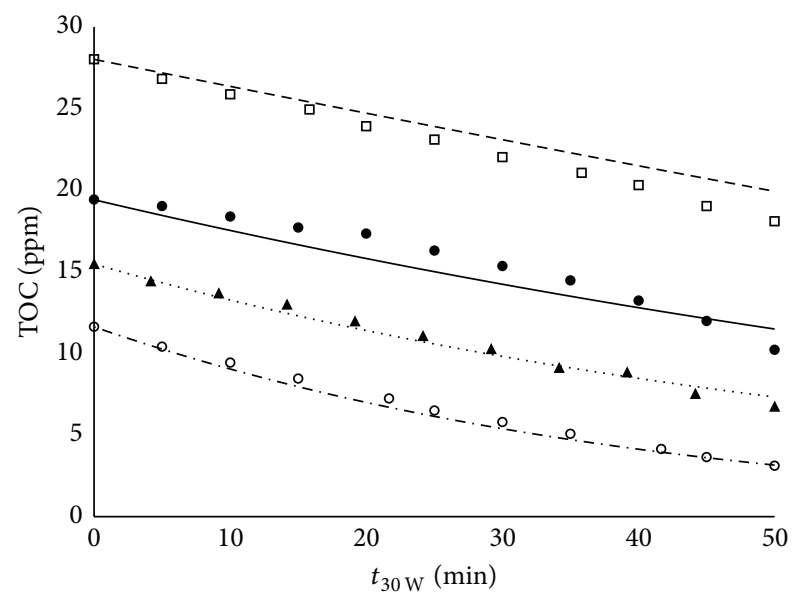

Figure 7: Solar mineralization of binary mixtures at $60 \mathrm{ppm}$.

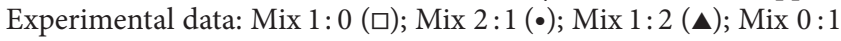
(O). Predictions: Mix 1:0 (---), Mix 2:1(-), Mix 1:2 (..), and Mix $0: 1(-\cdot)$, wherein "Mix" represents 4 -CP : DCA relation.

TABLE 3: Fitting statistical parameters for binary mixtures.

\begin{tabular}{lcccccc}
\hline \multicolumn{5}{c}{ Global concentration } \\
Parameter & \multicolumn{5}{c}{$30 \mathrm{ppm}$} & \multicolumn{2}{c}{$60 \mathrm{ppm}$} & \multicolumn{2}{c}{$120 \mathrm{ppm}$} \\
\multicolumn{1}{c}{ Mix 1:2 } & Mix 2:1 & Mix 1:2 & Mix 2:1 & Mix 1:2 & Mix 2:1 \\
\hline$R^{2}$ & -0.628 & 0.882 & 0.984 & 0.891 & 0.745 & 0.685 \\
$\sigma$ & 2.58 & 0.76 & 0.39 & 1.06 & 2.18 & 1.48 \\
\hline
\end{tabular}

The abovementioned was a deflection of the ideal behavior, which was not considered in the derivation of the proposed model and was likely due to the different interactions between reagents and intermediates. Also it could be caused by the $\mathrm{pH}$ decrease that produces positive aspects in the heterogeneous photodegradation of some organic compounds; 


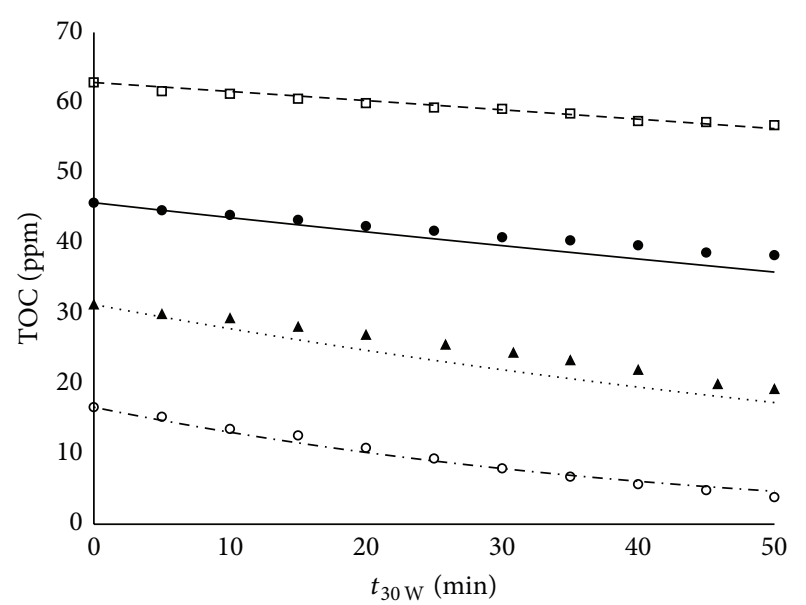

Figure 8: Solar mineralization of binary mixtures at $120 \mathrm{ppm}$.

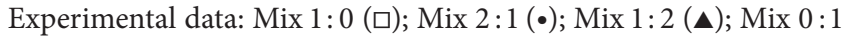
(O). Predictions: Mix 1:0 (- - ), Mix 2:1(—), Mix 1:2 (..), and Mix $0: 1(-\cdot)$, wherein "Mix" represents 4-CP :DCA relation.

it modifies the aggregation degree of the catalyst particles, at the same time influencing the molecules adsorption, light scattering and photon absorption, and ions movement among others [41].

The complexity of the degradation pathways, 4-CP (indirect attack by hydroxyl radicals absorbed), added to the DCA (direct attack by photogenerated holes), suggests a new route of degradation which can only assume that these attacks are still valid for each compound in the mixture.

This seems evident in the accurate prediction made by the proposed mixture model; however the appearance of the synergistic effect is a change or modification of the route of degradation. This could be caused by the occurrence of electrostatic surface charges of the catalyst (electrostatic attraction) caused by a decrease in $\mathrm{pH}$ and low concentration of substrate (formation of monolayers).

In general, the results suggest that it is possible to separate the total kinetic effect caused by the mixture, in partial kinetic contributions which are proportional to the concentration of each component in the mixture, as if each reagent was being oxidized independently under the same reaction conditions.

\section{Conclusions}

The proposed mixing rules yielded satisfactory results in the prediction of the heterogeneous solar photodegradation of binary mixtures using $\mathrm{TiO}_{2}-\mathrm{P} 25$. The kinetic behavior of the mixture is the result of individual kinetic contributions which are proportional to the effective concentration of TOC for the component in the mixture. The proposed mixing rules may be used in designing and scaling of multicomponent photocatalytic heterogeneous systems, considering that such rules may be extended to the use of intrinsic kinetic parameters. Also, radiant field effects do not affect the formulation of the mixing rules.

\section{Conflict of Interests}

The authors declare that there is no conflict of interests regarding the publication of this paper.

\section{Acknowledgments}

The authors thank the Vicerectoria de Investigaciones de Universidad del Valle and the Cartagena University for financing this publication. The authors thank COLCIENCIAS for funding doctoral studies.

\section{References}

[1] F. Arias, E. Ortiz, A. López-Vásquez, J. Colina-Márquez, and F. Machuca, "Photocatalytic decolonization of methylene blue with two photoreactors," Journal of Advanced Oxidation Technologies, vol. 11, no. 1, pp. 33-41, 2008.

[2] M. A. Mueses, F. Machuca-Martinez, and G. Li Puma, "Effective quantum yield and reaction rate model for evaluation of photocatalytic degradation of water contaminants in heterogeneous pilot-scale solar photoreactors," Chemical Engineering Journal, vol. 215-216, pp. 937-947, 2013.

[3] N. Suaterna-Ortíz, C. Insignares-Rendón, M. Mueses, and R. Camargo, "Pilot scale heterogeneous solar photodegradation of 4-chlorophenol in a cylindrical parabolic composed reactor (CPC)," Informacion Tecnologica, vol. 23, no. 6, pp. 13-24, 2012.

[4] J. Colina-Márquez, D. Díaz, A. Rendón, A. López-Vásquez, and F. Machuca-Martínez, "Photocatalytic treatment of a dye polluted industrial effluent with a solar pilot-scale CPC reactor," Journal of Advanced Oxidation Technologies, vol. 12, no. 1, pp. 93-99, 2009.

[5] J. Colina-Márquez, F. Machuca-Martínez, and G. Li Puma, "Photocatalytic mineralization of commercial herbicides in a pilot-scale solar CPC reactor: photoreactor modeling and reaction kinetics constants independent of radiation field," Environmental Science and Technology, vol. 43, no. 23, pp. 89538960, 2009.

[6] J. Colina-Márquez, Aplicabilidad del la Fotocatálisis Heterogénea Como Alternativa Complementaria en el Acople con un Sistema Biológico para la Eliminación de Pesticidas, Universidad del Valle, Cali, Colombia, 2010.

[7] S. Malato, P. Fernández-Ibáñez, M. I. Maldonado, J. Blanco, and W. Gernjak, "Decontamination and disinfection of water by solar photocatalysis: recent overview and trends," Catalysis Today, vol. 147, no. 1, pp. 1-59, 2009.

[8] S. Malato, J. Blanco, A. R. Fernández-Alba, and A. Agüera, "Solar photocatalytic mineralization of commercial pesticides: acrinathrin," Chemosphere, vol. 40, no. 4, pp. 403-409, 2000.

[9] C. Minero, E. Pelizzetti, S. Malato, and J. Blanco, "Large solar plant photocatalytic water decontamination: degradation of atrazine," Solar Energy, vol. 56, no. 5, pp. 411-419, 1996.

[10] J. Colina-Márquez and D. Castilla-Caballero, "Photocatalytic mineralization of wastewater polluted with commercial dicloxacillin in a pilot-scale solar CPC reactor," Ingeniería y Competitividad, vol. 15, no. 1, pp. 161-169, 2013.

[11] L. Elsellami, F. Vocanson, F. Dappozze et al., "Kinetic of adsorption and of photocatalytic degradation of phenylalanine effect of $\mathrm{pH}$ and light intensity," Applied Catalysis A: General, vol. 380, no. 1-2, pp. 142-148, 2010. 
[12] A. Gora, B. Toepfer, V. Puddu, and G. Li Puma, "Photocatalytic oxidation of herbicides in single-component and multicomponent systems: Reaction kinetics analysis," Applied Catalysis B: Environmental, vol. 65, no. 1-2, pp. 1-10, 2006.

[13] C. S. Zalazar, M. D. Labas, R. J. Brandi, and A. E. Cassano, "Dichloroacetic acid degradation employing hydrogen peroxide and UV radiation," Chemosphere, vol. 66, no. 5, pp. 808-815, 2007.

[14] F. Moulis and J. Krýsa, "Photocatalytic degradation of several VOCs (n-hexane, n-butyl acetate and toluene) on $\mathrm{TiO}_{2}$ layer in a closed-loop reactor," Catalysis Today, vol. 209, pp. 153-158, 2013.

[15] F. Mazille, T. Schoettl, N. Klamerth, S. Malato, and C. Pulgarin, "Field solar degradation of pesticides and emerging water contaminants mediated by polymer films containing titanium and iron oxide with synergistic heterogeneous photocatalytic activity at neutral pH," Water Research, vol. 44, no. 10, pp. 30293038, 2010.

[16] B. Toepfer, A. Gora, and G. L. Puma, "Photocatalytic oxidation of multicomponent solutions of herbicides: reaction kinetics analysis with explicit photon absorption effects," Applied Catalysis B: Environmental, vol. 68, pp. 171-180, 2006.

[17] J. Giménez, D. Curcó, and M. A. Queral, "Photocatalytic treatment of phenol and 2,4-dichlorophenol in a solar plant in the way to scaling-up," Catalysis Today, vol. 54, no. 2-3, pp. 229-243, 1999.

[18] D. Curcó, S. Malato, J. Blanco, and J. Giménez, "Photocatalysis and radiation absorption in a solar plant," Solar Energy Materials and Solar Cells, vol. 44, no. 2, pp. 199-217, 1996.

[19] D. Curcó, S. Malato, J. Blanco, J. Giménez, and P. Marco, "Photocatalytic degradation of phenol: comparison between pilot-plant-scale and laboratory results," Solar Energy, vol. 56, no. 5, pp. 387-400, 1996.

[20] F. Machuca, J. Colina-Márquez, and M. Mueses, "Determination of quantum yield in a heterogeneous photocatalytic system using a fitting-parameters model," Journal of Advanced Oxidation Technologies, vol. 11, no. 1, pp. 42-48, 2008.

[21] N. P. Xekoukoulotakis, C. Drosou, C. Brebou et al., "Kinetics of $\mathrm{UV}-\mathrm{A} / \mathrm{TiO}_{2}$ photocatalytic degradation and mineralization of the antibiotic sulfamethoxazole in aqueous matrices," Catalysis Today, vol. 161, no. 1, pp. 163-168, 2011.

[22] L. A. Ioannou, E. Hapeshi, M. I. Vasquez, D. Mantzavinos, and D. Fatta-Kassinos, "Solar/ $/ \mathrm{TiO}_{2}$ photocatalytic decomposition of $\beta$-blockers atenolol and propranolol in water and wastewater," Solar Energy, vol. 85, no. 9, pp. 1915-1926, 2011.

[23] J. Radjenovic, A. Bagastyo, R. A. Rozendal, Y. Mu, J. Keller, and K. Rabaey, "Electrochemical oxidation of trace organic contaminants in reverse osmosis concentrate using $\mathrm{RuO}_{2} / \mathrm{IrO}_{2}$ coated titanium anodes," Water Research, vol. 45, no. 4, pp. 1579-1586, 2011.

[24] R. Vargas and O. Núñez, "Photocatalytic degradation of oil industry hydrocarbons models at laboratory and at pilot-plant scale," Solar Energy, vol. 84, no. 2, pp. 345-351, 2010.

[25] E. Hapeshi, A. Achilleos, M. I. Vasquez et al., "Drugs degrading photocatalytically: kinetics and mechanisms of ofloxacin and atenolol removal on titania suspensions," Water Research, vol. 44, no. 6, pp. 1737-1746, 2010.

[26] V. Kitsiou, N. Filippidis, D. Mantzavinos, and I. Poulios, "Heterogeneous and homogeneous photocatalytic degradation of the insecticide imidacloprid in aqueous solutions," Applied Catalysis B: Environmental, vol. 86, no. 1-2, pp. 27-35, 2009.

[27] M. N. Abellán, J. Giménez, and S. Esplugas, "Photocatalytic degradation of antibiotics: the case of sulfamethoxazole and trimethoprim," Catalysis Today, vol. 144, no. 1-2, pp. 131-136, 2009.

[28] V. Augugliaro, V. Loddo, G. Marcì, L. Palmisano, and M. J. López-Muñoz, "Photocatalytic oxidation of cyanides in aqueous titanium dioxide suspensions," Journal of Catalysis, vol. 166, no. 2, pp. 272-283, 1997.

[29] J. Santiago-Morales, A. Agüera, M. D. M. Gómez et al., “Transformation products and reaction kinetics in simulated solar light photocatalytic degradation of propranolol using Ce-doped $\mathrm{TiO}_{2}$," Applied Catalysis B: Environmental, vol. 129, pp. 13-29, 2013.

[30] M. L. Mariani, R. J. Brandi, A. E. Cassano, and C. S. Zalazar, "A kinetic model for the degradation of dichloroacetic acid and formic acid in water employing the $\mathrm{H}_{2} \mathrm{O}_{2}$ /UV process," Chemical Engineering Journal, vol. 225, pp. 423-432, 2013.

[31] M. E. Lovato, C. A. Martín, and A. E. Cassano, "A reactionreactor model for $\mathrm{O}_{3}$ and UVC radiation degradation of dichloroacetic acid: the kinetics of three parallel reactions," Chemical Engineering Journal, vol. 171, no. 2, pp. 474-489, 2011.

[32] L. Rizzo, A. Della Sala, A. Fiorentino, and G. Li Puma, "Disinfection of urban wastewater by solar driven and UV lamp $\mathrm{TiO}_{2}$ photocatalysis: effect on a multi drug resistant Escherichia coli strain," Water Research, vol. 53, pp. 145-152, 2014.

[33] C. S. Zalazar, C. A. Martin, and A. E. Cassano, "Photocatalytic intrinsic reaction kinetics. II: effects of oxygen concentration on the kinetics of the photocatalytic degradation of dichloroacetic acid," Chemical Engineering Science, vol. 60, no. 15, pp. 43114322, 2005.

[34] N. C. Castillo, L. Ding, A. Heel, T. Graule, and C. Pulgarin, "On the photocatalytic degradation of phenol and dichloroacetate by $\mathrm{BiVO}_{4}$ : the need of a sacrificial electron acceptor," Journal of Photochemistry and Photobiology A: Chemistry, vol. 216, no. 2-3, pp. 221-227, 2010.

[35] M. L. Satuf, R. J. Brandi, A. E. Cassano, and O. M. Alfano, "Photocatalytic degradation of 4-chlorophenol: a kinetic study," Applied Catalysis B: Environmental, vol. 82, no. 1-2, pp. 37-49, 2008.

[36] J. A. Rengifo-Herrera, K. Pierzchała, A. Sienkiewicz, L. Forró, J. Kiwi, and C. Pulgarin, "Abatement of organics and Escherichia coli by $\mathrm{N}, \mathrm{S}$ co-doped $\mathrm{TiO}_{2}$ under $\mathrm{UV}$ and visible light. Implications of the formation of singlet oxygen $\left({ }^{1} \mathrm{O}_{2}\right)$ under visible light," Applied Catalysis B: Environmental, vol. 88, no. 34, pp. 398-406, 2009.

[37] O. M. Alfano, M. I. Cabrera, and A. E. Cassano, "Photocatalytic reactions involving hydroxyl radical attack: I. Reaction kinetics formulation with explicit photon absorption effects," Journal of Catalysis, vol. 172, no. 2, pp. 370-379, 1997.

[38] J. Cornell, Experiments With Mixtures: Designs, Models and the Analysis of Mixture Data, John Wiley \& Sons, New York, NY, USA, 1990.

[39] J. C. Lagarias, J. A. Reeds, M. Wright, and P. E. Wright, "Convergence properties of the Nelder-Mead simplex method in low dimensions," SIAM Journal on Optimization, vol. 9, no. 1, pp. 112-147, 1998.

[40] P. Harriott, Chemical Reactor Design, Marcel Dekker, New York, NY, USA, 1st edition, 2003.

[41] D. Friedmann, C. Mendive, and D. Bahnemann, " $\mathrm{TiO}_{2}$ for water treatment: parameters affecting the kinetics and mechanisms of photocatalysis," Applied Catalysis B: Environmental, vol. 99, no. 3-4, pp. 398-406, 2010. 

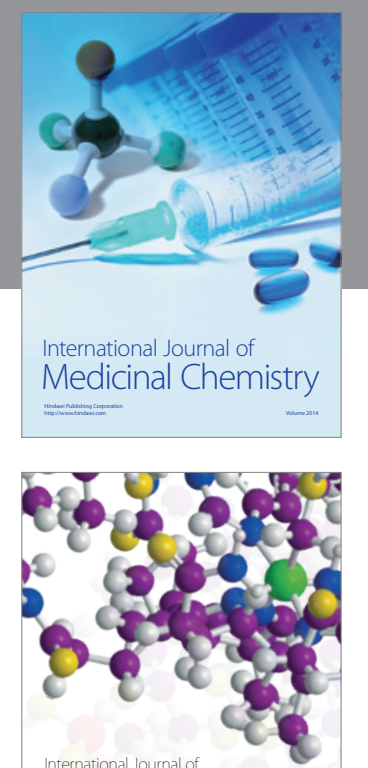

\section{Carbohydrate} Chemistry

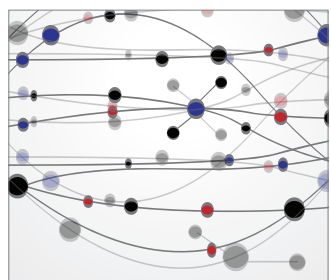

The Scientific World Journal
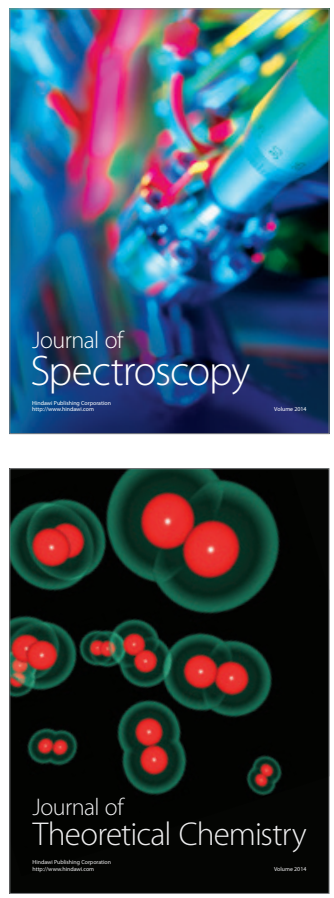
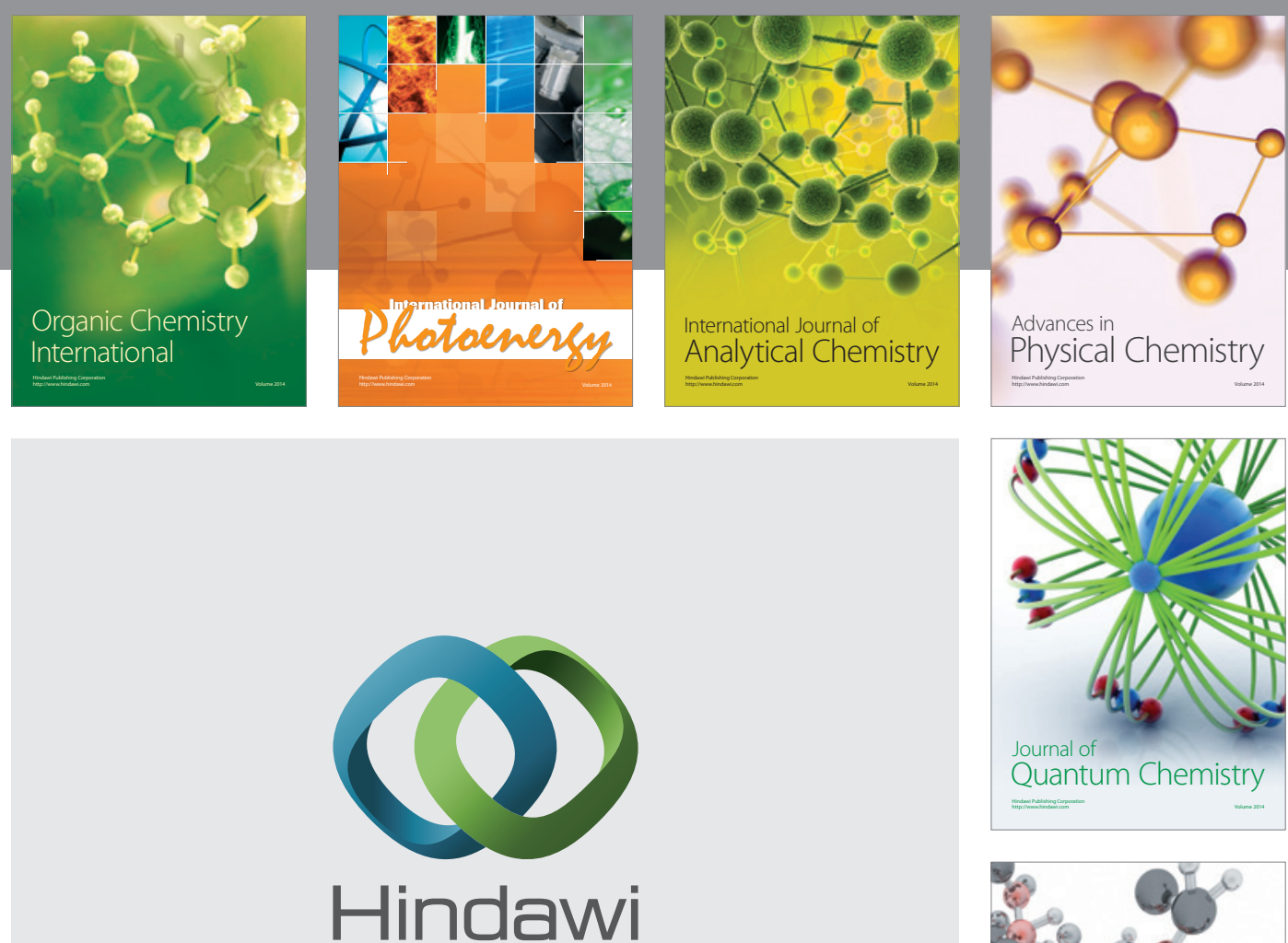

Submit your manuscripts at

http://www.hindawi.com

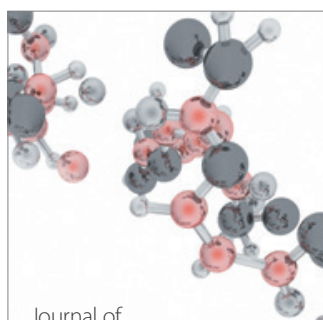

Analytical Methods

in Chemistry

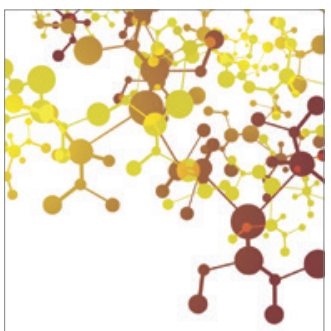

Journal of

Applied Chemistry

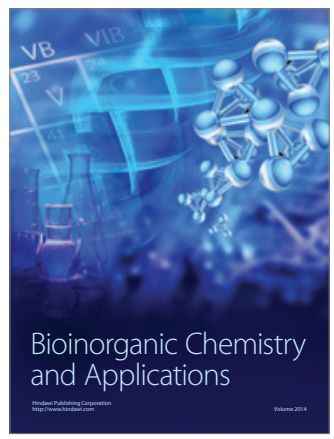

Inorganic Chemistry
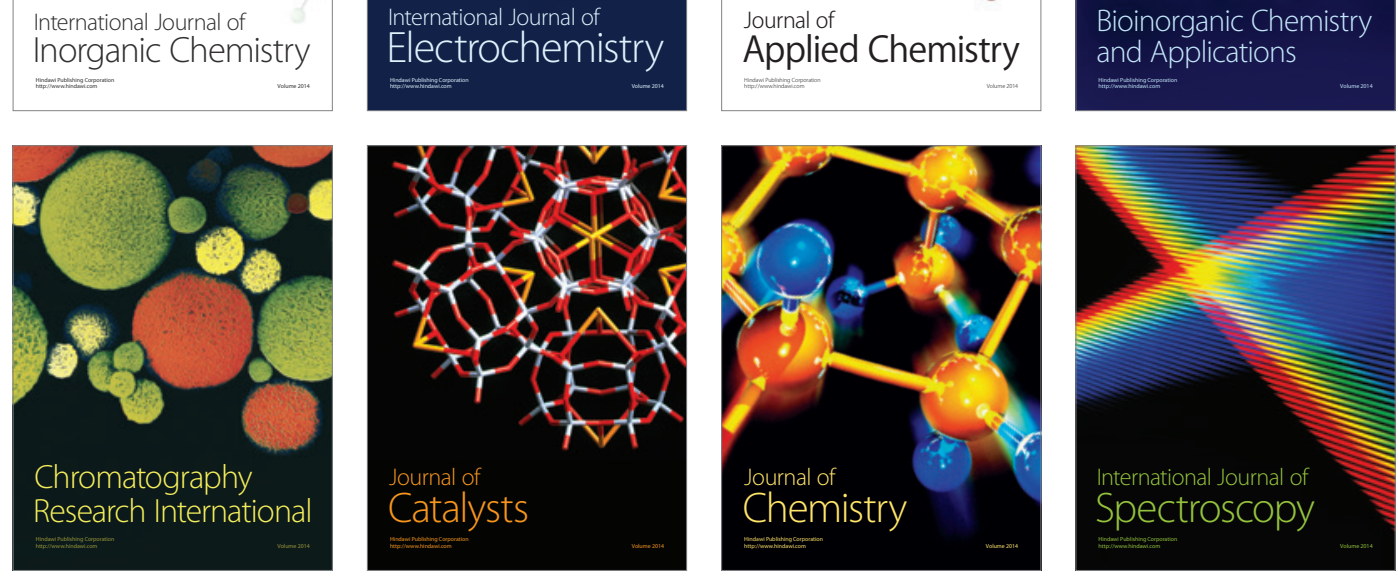